\title{
Study of coupled effect of impingement jet cooling of kerosene with solid structure
}

\author{
DU MengMeng ${ }^{1,2}$, ZHONG FengQuan $^{1,2^{*}}$, XING YunFei $^{1} \&$ ZHANG XinYu ${ }^{1,2}$ \\ ${ }^{1}$ State Key Laboratory of High Temperature Gas Dynamics, Institute of Mechanics, Chinese Academy of Sciences, Beijing 100190, China; \\ ${ }^{2}$ School of Engineering Science, University of Chinese Academy of Sciences, Beijing 100049, China
}

Received December 20, 2018; accepted May 5, 2019; published online August 30, 2019

\begin{abstract}
Characteristics of flow and heat transfer of kerosene impingement jets were studied numerically. The coupled effect of heat transfer of fluid and structure was investigated. Numerical simulation of fluid flow shows that compared to convective heat transfer of kerosene flow in cooling channels, impingement jet cooling significantly enhances heat transfer ability. At the same time, the pressure loss is below one atmospheric pressure. Both stress and strain of high temperature nickle-based alloy structure were analyzed with typical thermal loading and impingement cooling effect. The numerical results show that temperature distribution in the hot surface of the solid structure is relatively uniform and far below the maximum allowable temperature of the alloy material. The strength analysis shows that both stress and strain of the solid structure meet the material requirements.
\end{abstract}

impingement jet cooling, hydrocarbon fuel, heat transfer enhancement, fluid-solid coupling

Citation: Du M M, Zhong F Q, Xing Y F, et al. Study of coupled effect of impingement jet cooling of kerosene with solid structure. Sci China Tech Sci, 2019, 62: 2021-2028, https://doi.org/10.1007/s11431-018-9521-1

\section{Introduction}

Impingement jet cooling technique is an effective cooling method with high heat transfer coefficient, and it has been widely used in many engineering applications including cooling for gas turbine combustors, electronic components and metal processing [1-3]. The coolant flow forms highspeed jets and strong vortex structures through multiple jet holes, and conducts efficient heat transfer for the impacted wall plate. This cooling method is especially suitable for cooling of local region with very high wall heat flux.

Literatures of properties of impingement heat transfer of simple fluids such as air, water are very extensive. Kiper [4] carried out an experimental study of performance of water impingement jet cooling. Aldabbagh et al. [5] numerically studied flow field of air impingement jets and distribution of heat transfer coefficient. Katti et al. [6] studied the re-

*Corresponding author (email: fzhong@imech.ac.cn) lationship between the secondary peak phenomenon of heat transfer coefficient and Reynolds number of impingement jet. Hollworth et al. [7], Behbahani et al. [8] studied influences of structural parameters on heat transfer of air impingement jet with constant flow rate. Qin et al. [9], Fabbri et al. [10] studied heat transfer properties of liquid jet such as deionized water and FC40. However, to the author's knowledge, studies about impinging jet cooling of complicated fluid such as hydrocarbon fuels are very few. It is known that hydrocarbon fuels can be used as coolant for active thermal protection of engine systems [11,12]. Hydrocarbon fuels, such as aviation kerosene, have complex thermal properties and multi-state changes (liquid, supercritical and gaseous state) and their thermodynamic and transport parameters present dramatic variations near the critical point $[13,14]$. Thus, cooling performance of impinging jets of hydrocarbon fuel is much more complicated than that of air or water. It is imperative to study flow and heat transfer characteristics of kerosene impingement jet 
cooling.

At the same time, thermal protection not only requires high heat transfer ability to keep the structure temperature within the allowable working temperature range of solid material, but also needs thermal stress and strain of the cooled structure to meet the strength requirement. Large thermal stress could cause structural failure and large strain could reduce the service life of the material. Therefore, for a complete study of impingement jet cooling effect, both heat transfer performance and structural strength need to be investigated. In this paper, flow and heat transfer of impingement jet cooling of aviation kerosene are studied numerically, and the flow-solid coupled heat transfer properties is studied and the strength performance of the cooled structure is analyzed.

\section{Computational models and methods}

\subsection{Computational models}

The geometry of impingement jet cooling is shown in Figure 1. The kerosene coolant flows into plenum from the pipe inlet, and then it impinges on the cooling surface through multiple holes, and then flows out of the cooling structure through the collecting groove and to the pipe outlet. There are twelve evenly distributed circular impingement jet holes with $1 \mathrm{~mm}$ in diameter. The jet-to-jet spacing of holes is $8 \mathrm{~mm}$, and the distance from jet exit to the impingement cooling plate is $3 \mathrm{~mm}$.

Figure 2 shows the solid model of the impingement jets structure. The coolant jets impact on the cooling surface of the impingement cooling plate with a thickness of $1.5 \mathrm{~mm}$. As shown in the Figure 2, the bottom surface of the impingement cooling plate is the heating surface with high heat flux loaded on it and it is also called as hot wall.

\subsection{Computational methods}

In this paper, the sequential coupling method is used to simulate the interaction between fluid domain and solid domain. First, the governing equation of flow is solved without taking account of the influence of structural deformation, and the heat transfer coefficient distribution of the impingement cooling surface is obtained. The result of heat transfer coefficient and the coolant temperature loaded on the cold surface of the solid structure are used as the thermal boundary conditions to calculate the solid temperature. Finally, stress and strain fields of solid structure under the combined action of thermal load and impingement cooling effect are analyzed. This sequential coupling method has been applied to many flow-solid coupling problems $[15,16]$, especially for those with very little structural deformation that has negligible effects on flow.

For simulation of flow and heat transfer of the impinging
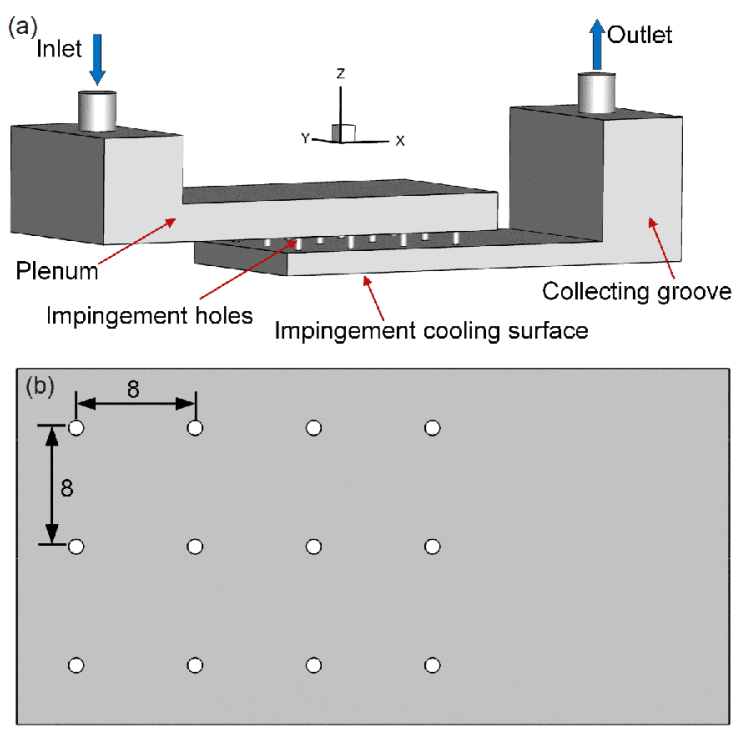

Figure 1 (Color online) Impingement jet cooling model. (a) Fluid computational domain; (b) schematic of impingement holes distributions (unit: $\mathrm{mm})$.

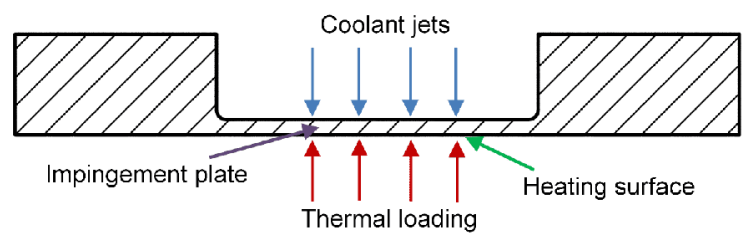

Figure 2 (Color online) Schematic of impingement jet structure.

jet cooling, Navier-Stokes (N-S) equation is solved by Reynolds-average method, and the turbulence is simulated with SST $k-\omega$ model. Meanwhile, the convection term is discretized by a second-order upwind scheme, and the diffusion term is discretized by a second-order central scheme. Stretch mesh was adopted near the wall boundaries and make sure the minimum grid scale $\left(\Delta y^{+}\right) \sim 1$. The thermal condition of cooling surface is set as isothermal boundary with temperature of $800 \mathrm{~K}$. The heat flux distribution of the cooling surface is obtained by solving the N-S equation, and then the heat transfer coefficient distribution of the impingement cooling surface is obtained by its definition of $h=q_{\mathrm{w}} /\left(T_{\mathrm{w}}-T_{\mathrm{f}}\right)$, where $q_{\mathrm{w}}$ is the heat flux rate, $T_{\mathrm{w}}$ is the wall temperature, and $T_{\mathrm{f}}$ is the inlet temperature of coolant. In this paper, flow and heat transfer characteristics of kerosene impingement jet cooling with different inlet temperatures are numerically studied. The heat transfer coefficient and the pressure loss through the cooling structure are also studied. The thermal properties of kerosene are determined with a 10 -species surrogate proposed by Zhong et al. [14]. The critical temperature of kerosene is about $650 \mathrm{~K}$ and the critical pressure is $2.2 \mathrm{MPa}$. When the kerosene pressure is higher than the critical value, as the temperature increases, kerosene can directly change from liquid state to supercritical state with- 
out two-phase coexistence. Figure 3 shows the thermal properties of kerosene as a function of temperature at $3 \mathrm{MPa}$. It can be seen that density and viscosity of kerosene decrease dramatically near the critical point and specific heat has a peak value near it.

For simulation of temperature field, stress and strain of the solid structure, finite element method is used. The nonlinear effect of material mechanical properties is taken into account, and the yield criterion of material adopts Von Mises criterion. The total strain of the structure for small deformation is composed of elastic strain, plastic strain and thermal strain. The relationship between elastic strain and stress follows Hooke law, and the kinematic hardening model is used to determine the subsequent yield surface of material in the stage of plastic deformation $[17,18]$.

The material of the structure is nickle-based alloy GH3625, which has good mechanical properties even at high temperature of $1250 \mathrm{~K}$. Table 1 shows thermal and mechanical properties of the material as a function of temperature, and its detailed performance parameters can be found in ref. [19].

\subsection{Boundary conditions}

For analysis of flow and heat transfer, the mass flow rate of the coolant is $50 \mathrm{~g} / \mathrm{s}$, and the total pressure is $3 \mathrm{MPa}$. The inlet temperature of kerosene is 300 and $600 \mathrm{~K}$ for the two cases, and the corresponding impingement jet Reynolds number (defined according to the diameter and velocity of jet) is 4400 and 53100, respectively. As discussed in refs. [20,21], impingement jet flow is in turbulent state when Reynolds number is higher than 2000.

For thermal analysis of solid structure, in order to ensure the continuity of heat flow in the fluid-solid interface, the third boundary condition is given as

$h_{\mathrm{f}}=\left(T_{\mathrm{w}}-T_{\mathrm{f}}\right)=\left.\mathbf{n} \cdot \lambda \nabla T\right|_{\mathrm{w}}$,

where $h_{\mathrm{f}}$ is heat transfer coefficient, $T_{\mathrm{w}}$ is wall temperature, $\mathbf{n}$ is the normal vector of the wall, and $\lambda$ is thermal conductivity of the material.

A uniform heat flux with two values of $1,2 \mathrm{MW} / \mathrm{m}^{2}$ are imposed on the heating wall of the impingement structure respectively, and the other walls are adiabatic wall boundary. The high pressure of $3 \mathrm{MPa}$ of the kerosene flow is taken into account to analyze the strength of the structure.

\section{Results and discussion}

\subsection{Validation of numerical method of impingement jet flow}

The numerical method for flow and heat transfer is validated by comparing the calculated heat transfer coefficient with the experimental result by Xing et al. [22]. The distribution of non-dimensional heat transfer coefficient, Nusselt number, on the impingement cooling wall along the centerline is shown in Figure 4 with comparisons of the experimental data in ref. [22]. As shown in Figure 4, the calculated result is in good agreement with the experimental data, which indicates the accuracy of the present numerical method.
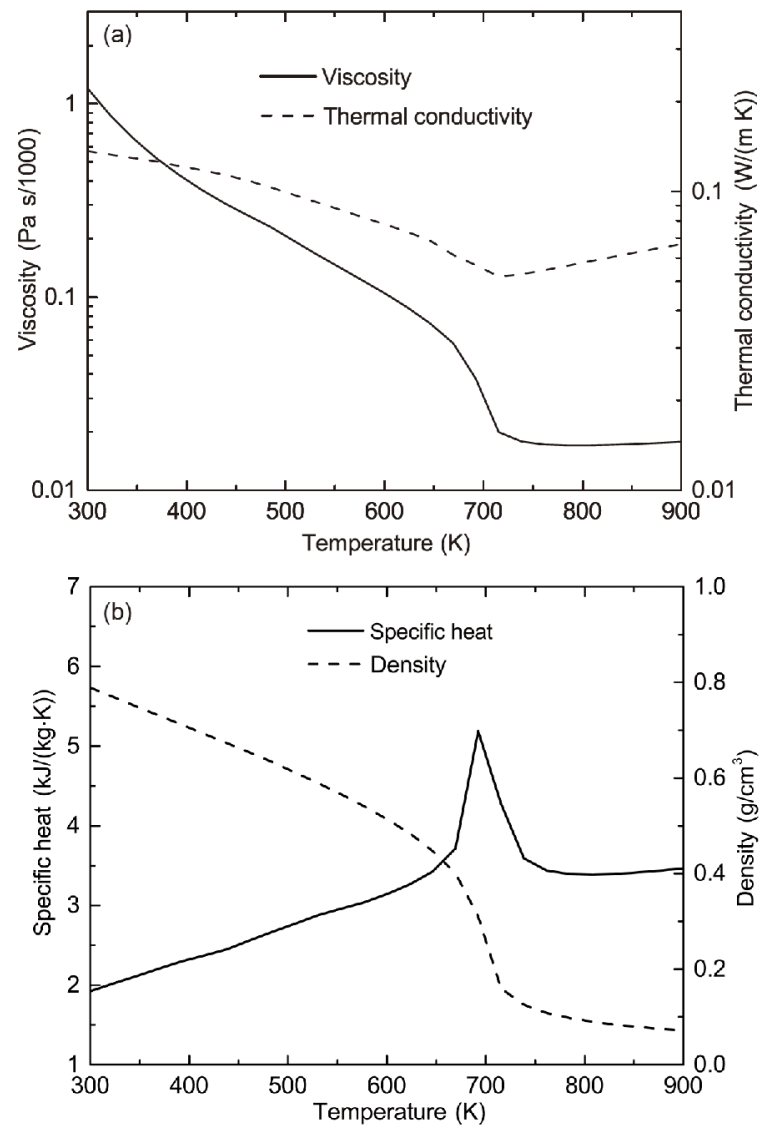

Figure 3 Properties of kerosene at $3 \mathrm{MPa}$. (a) Viscosity and thermal conductivity as a function of temperature; (b) specific heat and density as a function of temperature.

Table 1 Material's physical properties

\begin{tabular}{cccc}
\hline$T(\mathrm{~K})$ & $E(\mathrm{GPa})$ & $\mu$ & $k(\mathrm{~W} /(\mathrm{m} \mathrm{K}))$ \\
\hline 293 & 205 & 0.308 & 9.5 \\
368 & 200 & 0.310 & 10.9 \\
478 & 195 & 0.312 & 12.6 \\
588 & 190 & 0.313 & 14.2 \\
698 & 185 & 0.312 & 15.7 \\
813 & 175 & 0.321 & 17.8 \\
923 & 170 & 0.328 & 19.2 \\
1033 & 160 & 0.329 & 20.9 \\
1143 & 146 & - & 22.8 \\
\hline
\end{tabular}




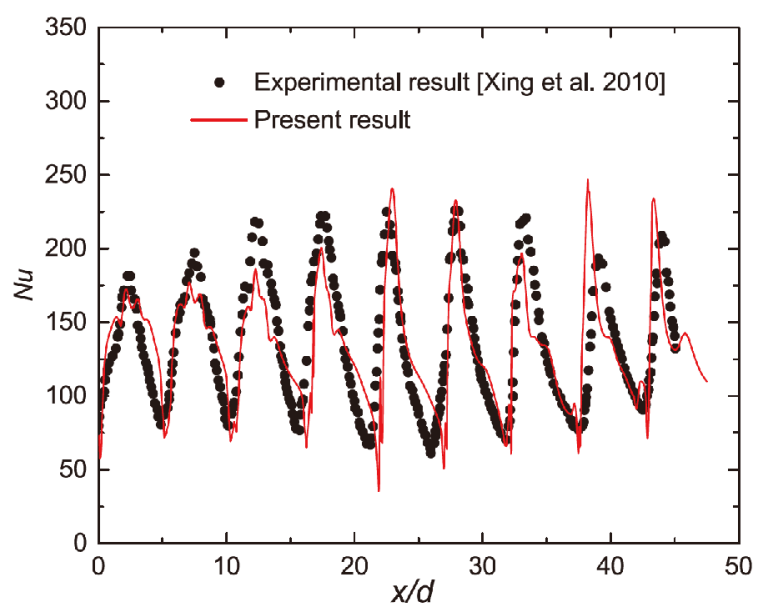

Figure 4 (Color online) Comparison between numerical result and experimental data.

\subsection{Study of mesh dependency}

The numerical results computed with five different mesh sizes with a total mesh number of $1.1,2.1,4.1,5.8,8.0$ million are compared. The kerosene mass flow rate is $50 \mathrm{~g} / \mathrm{s}$, and the inlet temperature and pressure is 300 and $3 \mathrm{MPa}$. Figure 5(a) and (b) shows the results of heat transfer coefficient and pressure loss for the five meshes. The heat transfer coefficient is the averaged value on the impingement cooling plate and the pressure loss is the pressure difference between the inlet and the outlet. As shown in the figures, the heat transfer coefficient and the pressure results are almost independent with mesh number when the mesh number is larger than 4.1 million. Hence, the mesh size with 5.8 million cells is used for the present simulation.

\subsection{Results of flow and heat transfer}

\subsubsection{Results with inlet temperature $300 \mathrm{~K}$}

Figure 6 shows result of kerosene impingement jets flow with an inlet temperature of $300 \mathrm{~K}$. The streamlines from side view and top view are shown in Figure 6(a) and (b), respectively. Streamlines of some longitudinal and cross sections through the holes are shown in Figure 6(c) and (d). As shown in Figure 6(a) and (b), kerosene coolant flows into the plenum and then accelerates through the holes to impinge on the cooling surface and generate multiple jets interacting each other. Typical counter rotating vortex pairs are formed in the impinging zone, and they entrain hot fluid near the wall into the main coolant flow and enhance the flow mixing, and therefore increases the heat transfer efficiency significantly.

Figure 7 shows distribution of heat transfer coefficient on the cooling surface. It can be seen that the value of heat transfer coefficient on the stagnation point of the jet (right against the holes) is highest, and the value far away from the
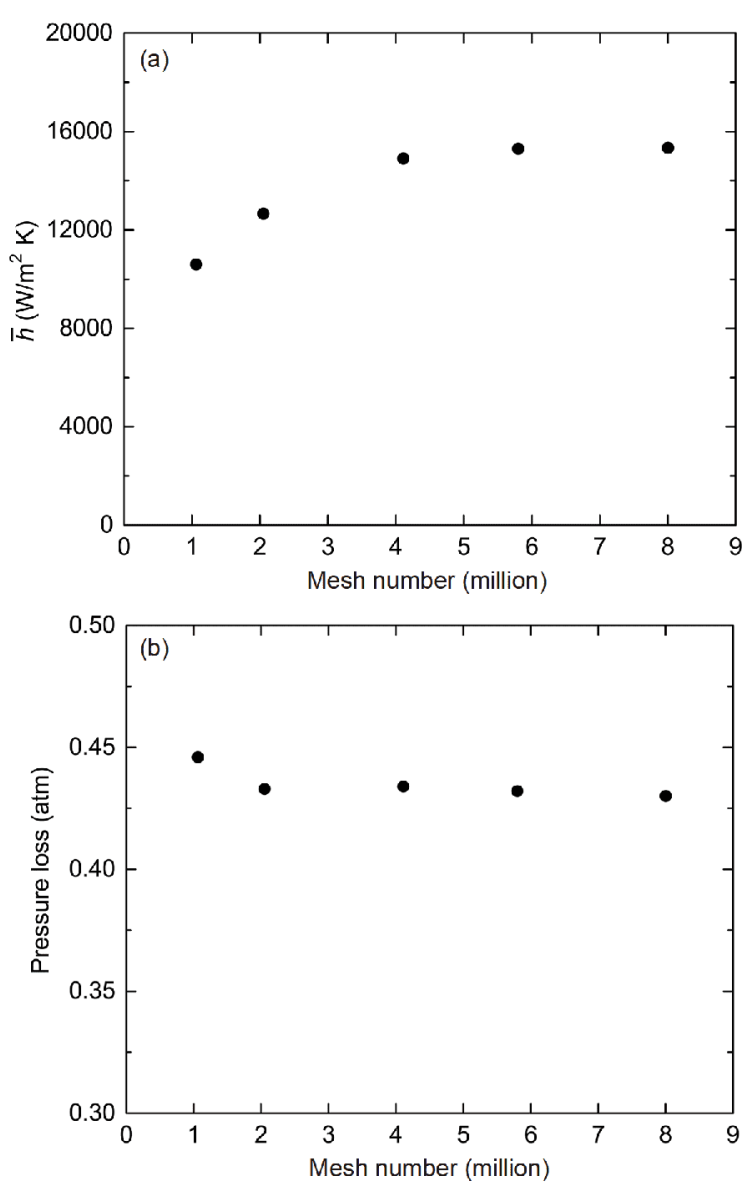

Figure 5 Results of mesh dependency study. (a) Heat transfer coefficient for different mesh sizes; (b) pressure loss for different mesh sizes.

stagnation point decreases rapidly. The heat transfer coefficient presents bell-shaped distribution with a peak in the regions right against the holes.

\subsubsection{Results with inlet temperature $600 \mathrm{~K}$}

When the inlet temperature of kerosene is $600 \mathrm{~K}$ that is close to the critical value. In the process of impingement jet cooling, kerosene transforms from liquid to supercritical state as its temperature increases.

The flow field at high temperature of $600 \mathrm{~K}$ is similar to that at low temperature of $300 \mathrm{~K}$ that multiple vortex structures exist. However, there are significant differences in the heat transfer coefficient distribution. As shown in Figure 8, the overall value of heat transfer coefficient at high temperature of $600 \mathrm{~K}$ is obviously higher than that at low temperature of $300 \mathrm{~K}$. More importantly, the peak of heat transfer coefficient is located in the vicinity of the stagnation point of each kerosene jet, instead of just on the stagnation point. For a relatively large ratio of impingement distance to the diameter of the impingement holes, the shifting of heat transfer peak with increased Reynolds number has been observed and discussed in the previous work with simple 


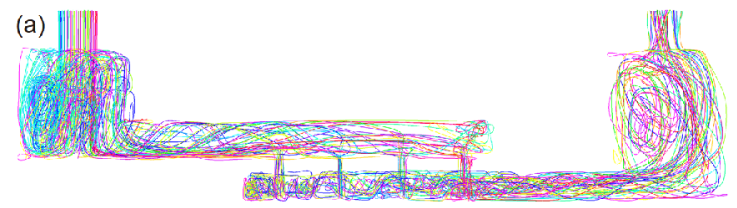

(c)

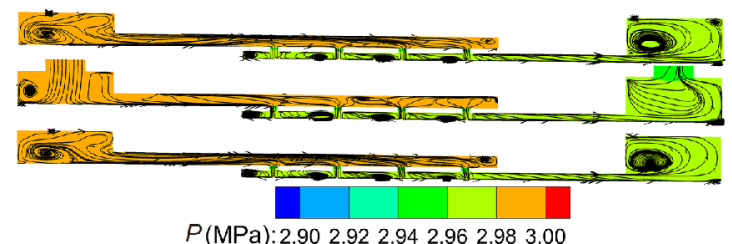

(b)

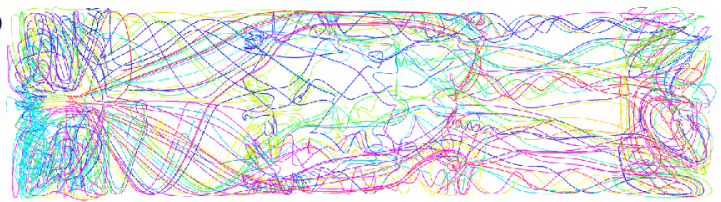

(d)

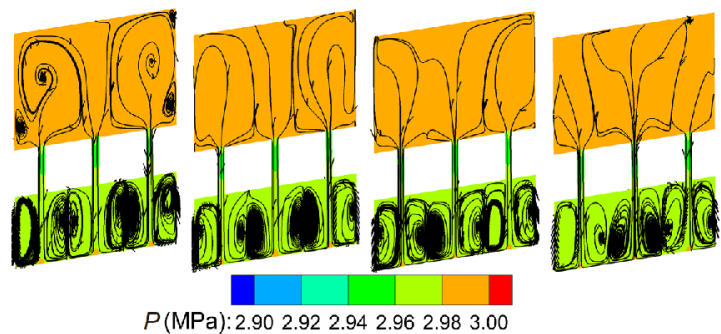

Figure 6 (Color online) Streamlines of impingement jet flow. (a) Volume streamlines (side view); (b) volume streamlines (top view); (c) streamlines of longitudinal sections; (d) streamlines of cross sections.

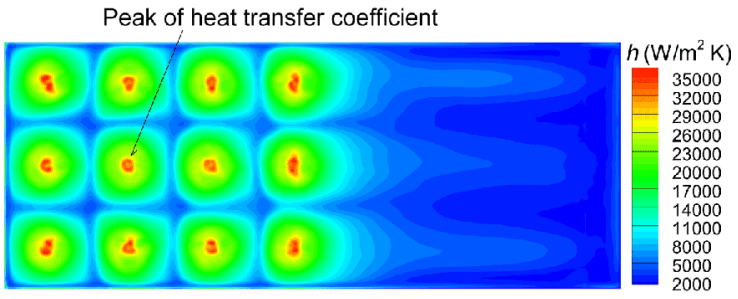

Figure 7 (Color online) Contours of heat transfer coefficient on the cooling surface with inlet temperature $300 \mathrm{~K}$.

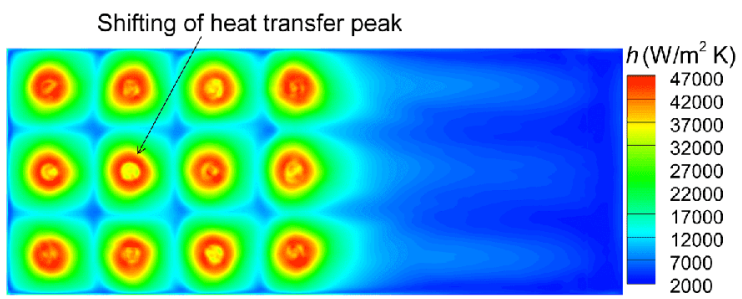

Figure 8 (Color online) Contours of heat transfer coefficient on the impingement cooling plate with inlet temperature $600 \mathrm{~K}$.

fluids such as air [23-25]. For the present study, the Reynolds number of impingement jets flow with a higher inlet temperature of $600 \mathrm{~K}$ is much larger than that with an inlet temperature of $300 \mathrm{~K}$ since viscosity of kerosene decreases significantly as inlet temperature increases. The shifting of heat transfer peak is believed to be caused by local flow acceleration due to relatively large impingement jet velocity as discussed in refs. [23-25].

\subsubsection{The heat transfer effectiveness of impingement jet cooling}

Table 2 shows the averaged heat transfer coefficient of impingement jet cooling surface and pressure loss between the inlet and the outlet. Table 2 also presents the results of heat transfer coefficient for four $2 \mathrm{~mm} \times 2 \mathrm{~mm}$ square cooling channels uniformly distributed along the spanwise direction of the cooling plate with the same kerosene mass flow rate. The convective heat transfer coefficient of square channel is obtained by classic Sieder-Tate heat transfer formula [26] for fully developed turbulent pipe flow channel. As shown in Table 2, for both cases, the heat transfer coefficient of impingement jet cooling is more than $60 \%$ higher than that of the cooling channels. The Table 2 indicates that the pressure loss $\Delta P$ is less than one atmosphere for both cases due to impingement jets.

\subsection{Simulation results of solid structure}

Four cases with different heat flux loading and inlet temperature of kerosene cooling are calculated for the cooled solid panel with a configuration indicated in Figure 2. Table 3 lists parameters for the four cases. Figure 9 shows the contours of temperature distribution on the hot wall of the solid structure by solving heat conduction equation.

As shown in Figure 9, temperature on the hot wall of the solid structure is significantly lower than the maximum working temperature of the material. When the heat flux is $1 \mathrm{MW} / \mathrm{m}^{2}$, the maximum temperature of the solid structure is only $759 \mathrm{~K}$ for case 2 with higher inlet coolant temperature. When the wall heat flux is $2 \mathrm{MW} / \mathrm{m}^{2}$, the maximum temperature is increased to $899 \mathrm{~K}$ for case 4 with higher inlet coolant temperature. The temperature distribution on the hot wall is relatively uniform due to three-dimensional heat conduction effect, and the root mean square (RMS) value of temperature variation is less than $24.0 \mathrm{~K}$ for all the cases.

Table 4 shows quantitative temperature result on the hot wall. It can be seen that the temperature increases with the increasing heat flux under the same coolant temperature.

For the case 1 and case 2, the maximum temperature of the solid structure is lower than case 3 and case 4 since the inlet temperature of kerosene for case 1 and case 2 is much lower than that of case 3 and case 4 . The RMS of temperature 
Table 2 Averaged cooling results

\begin{tabular}{ccccc}
\hline Inlet $T(\mathrm{~K})$ & $R e$ & Impingement jet cooling $\bar{h}\left(\mathrm{~W} /\left(\mathrm{m}^{2} \mathrm{~K}\right)\right)$ & Rectangular channel cooling $\bar{h}\left(\mathrm{~W} /\left(\mathrm{m}^{2} \mathrm{~K}\right)\right)$ & $\Delta P(\operatorname{atm})$ \\
\hline 300 & 4400 & 15300 & 6700 & 0.43 \\
600 & 53100 & 23719 & 14500 & 0.64 \\
\hline
\end{tabular}

Table 3 Parameters for the four cases

\begin{tabular}{ccccc}
\hline Case number & Kerosene mass flow rate $(\mathrm{g} / \mathrm{s})$ & Inlet pressure of kerosene $(\mathrm{MPa})$ & Inlet temperature of kerosene $(\mathrm{K})$ & Wall heat flux $\left(\mathrm{MW} / \mathrm{m}^{2}\right)$ \\
\hline 1 & 50 & 3 & 300 & 1 \\
2 & 50 & 3 & 300 & 2 \\
3 & 50 & 3 & 600 & 1 \\
4 & 50 & 3 & 600 & 2 \\
\hline
\end{tabular}
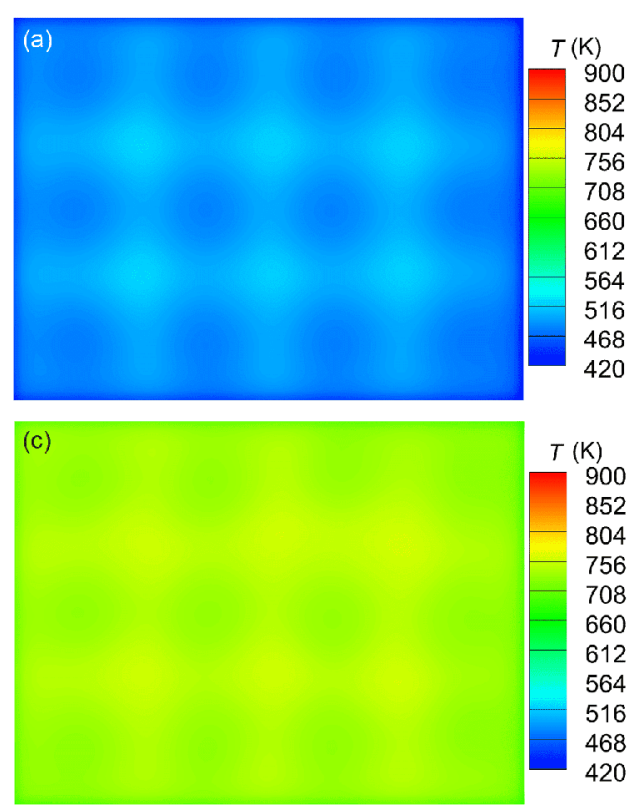
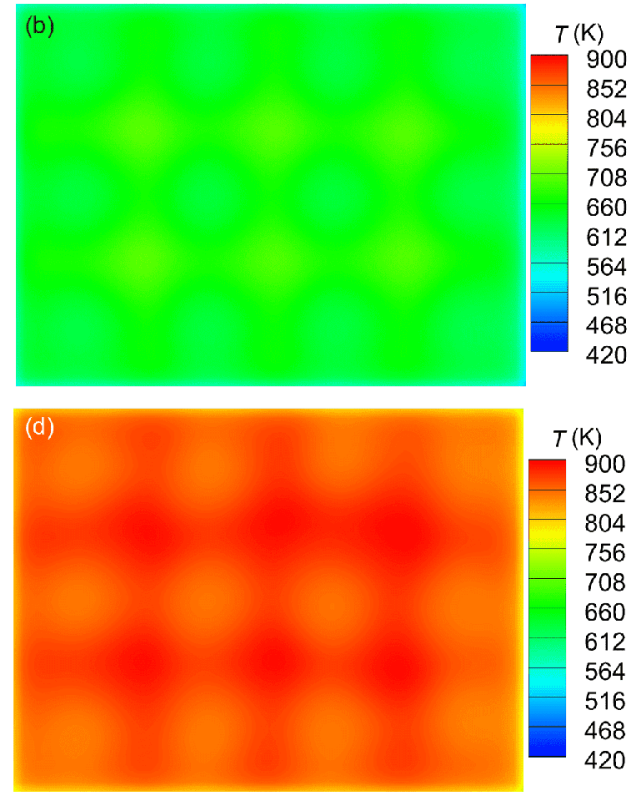

Figure 9 (Color online) Temperature distribution on the hot wall of impingement structure. (a) Surface temperature distributions of case 1; (b) surface temperature distributions of case 2; (c) surface temperature distributions of case 3; (d) surface temperature distributions of case 4.

Table 4 Temperature of the hot wall

\begin{tabular}{cccc}
\hline Case number & Maximum temperature of the hot wall $(\mathrm{K})$ & Average temperature of the hot wall $(\mathrm{K})$ & RMS of temperature variation $(\mathrm{K})$ \\
\hline 1 & 519.5 & 490.6 & 13.7 \\
2 & 701.7 & 650.2 & 24.0 \\
3 & 759.4 & 738.1 & 10.2 \\
4 & 899.2 & 860.3 & 18.4 \\
\hline
\end{tabular}

variations for the low heat flux of $1 \mathrm{MW} / \mathrm{m}^{2}$ is smaller than that for the high heat flux of $2 \mathrm{MW} / \mathrm{m}^{2}$. It attributes to that for low heat flux, the transverse heat conduction is decreased as well as the temperature gradient on the hot wall.

Figure 10 shows the distribution of Von Mises stress and equivalent plastic/elastic strain on the hot wall under the combined effect of thermal load and impingement jet cooling. The solid structure is in plastic state when the Von Mises stress exceeds the yield strength of high-temperature alloy, otherwise it is in elastic state. For case 1 and case 3, the temperature gradient of the solid structure is relatively low on account of the low heat flux, so the maximum stress is also low, which are 332.0 and $303.8 \mathrm{MPa}$, respectively for case 1 and case 3 , and the solid structures are both in the elastic stage. When the heat flux increases to $2 \mathrm{MW} / \mathrm{m}^{2}$ for case 2 and case 4 , the stress and strain of the structure also 
increase, and partial regions undergo plastic deformation slightly.

Table 5 shows strength results of the structure on the hot
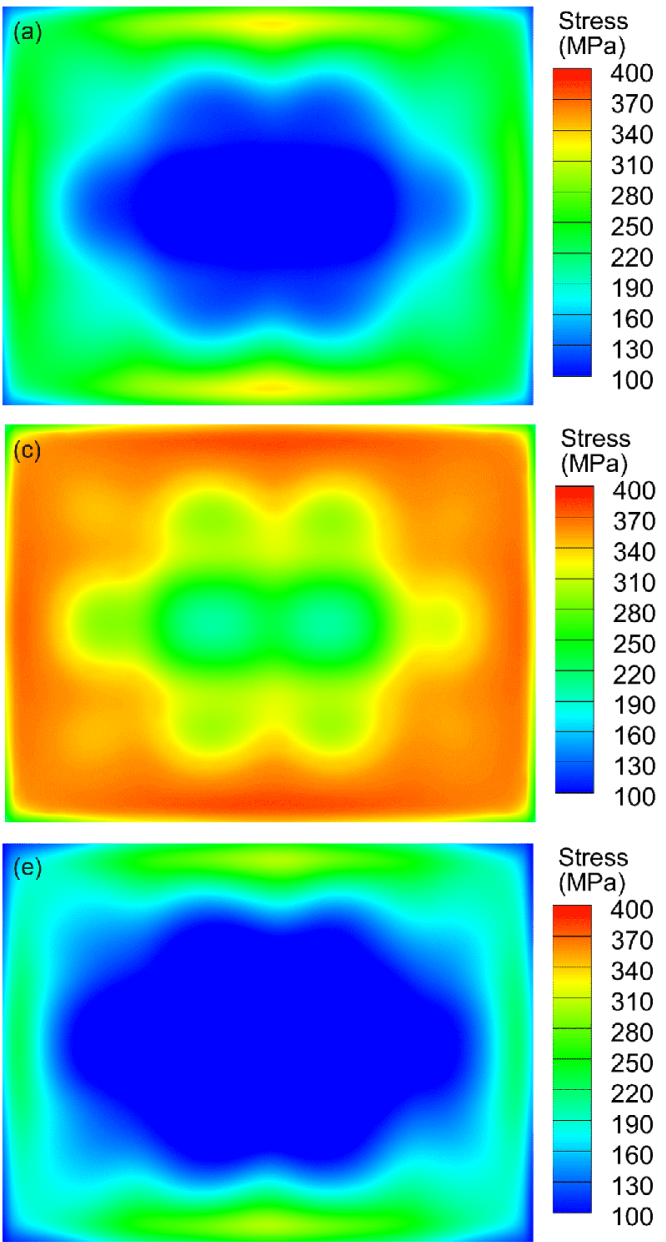

\section{Stress} (MPa) 400

370

340

310

280

250

220
190

160
130

100

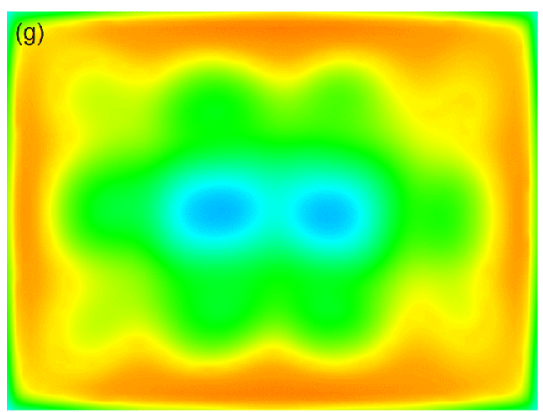

Stress (MPa)

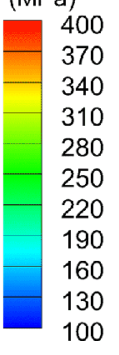

wall. It indicates that the maximum plastic strain is less than $0.12 \%$. Under the combined effect of thermal load and impingement jet cooling, the stress and strain of the impinge-
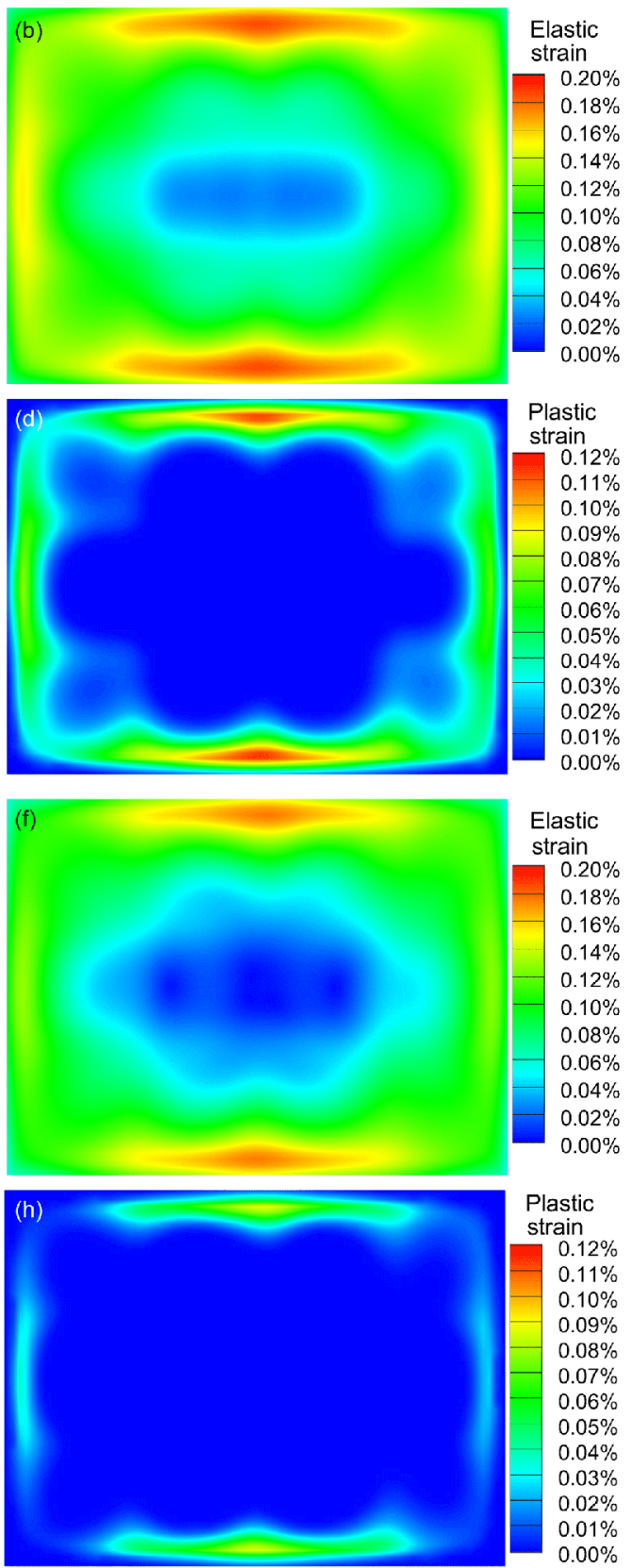

Figure 10 (Color online) Stress and strain distributions on the hot wall. (a) Stress distribution of case 1; (b) strain distribution of case 1; (c) stress distribution of case 2; (d) strain distribution of case 2; (e) stress distribution of case 3; (f) strain distribution of case 3; (g) stress distribution of case 4; (h) strain distribution of case 4 .

Table 5 Stress and strain

\begin{tabular}{|c|c|c|c|}
\hline Case number & Maximum stress (MPa) & Maximum plastic strain & Maximum elastic strain \\
\hline 1 & 332.0 & - & $0.186 \%$ \\
\hline 2 & 380.3 & $0.114 \%$ & - \\
\hline 3 & 303.8 & - & $0.177 \%$ \\
\hline 4 & 362.4 & $0.088 \%$ & - \\
\hline
\end{tabular}


ment structure as given in the paper can meet the strength requirements of high temperature alloy material.

\section{Conclusions}

In the present work, flow and heat transfer characteristics of impingement jet cooling of aviation kerosene are numerically studied. The coupling heat transfer and the strength of the cooled solid structure are analyzed. Several conclusions may be obtained.

(1) Impingement jets with high speed form significant vortex structures, which enhances mixing and heat transfer of kerosene flow. With increasing in the inlet temperature, Reynolds number of kerosene impingement jets is increased, which results in shifting of the peak of heat transfer coefficient from the stagnation point of jets to its vicinity.

(2) Impingement jet cooling of kerosene has been proven an efficient cooling method. The heat transfer coefficient is more than $60 \%$ higher than the traditional channel cooling method and the pressure loss is less than 1 atmosphere.

(3) The temperature of the solid structure with impingement jet cooling is much lower than the maximum working temperature of the alloy material, and the temperature distribution on the hot wall is relatively uniform with RMS of temperature variations less than $24 \mathrm{~K}$.

(4) The results of structure strength under the combined effect of thermal loading and impingement cooling show that stress and strain of the alloy structure are within the allowable range of the material.

This work was supported by the National Natural Science Foundation of China (Grant No. 11672307), and the Youth Innovation Promotion Association of the Chinese Academy of Sciences.

1 Taslim M E, Bethka D. Experimental and numerical impingement heat transfer in an airfoil leading-edge cooling channel with cross-flow. J Turbomach, 2009, 131: 011021

2 Chen S J, Tseng A A. Spray and jet cooling in steel rolling. Int J Heat Fluid Flow, 1992, 13: 358-369

3 Kondo Y, Matsushima H, Komatsu T. Optimization of pin-fin heat sinks for impingement cooling of electronic packages. J Electron Packag, 2000, 122: 240-246

4 Kiper A M. Impinging water jet cooling of VLSI circuits. Int Commun Heat Mass Transfer, 1984, 11: 517-526

5 Aldabbagh L B Y, Sezai I. Numerical simulation of three-dimensional laminar, square twin-jet impingement on a flat plate, flow structure, and heat transfer. Numer Heat Transfer Part A-Appl, 2002, 41: 835850

6 Katti V, Prabhu S V. Heat transfer enhancement on a flat surface with axisymmetric detached ribs by normal impingement of circular air jet.
Int J Heat Fluid Flow, 2008, 29: 1279-1294

7 Hollworth B R, Berry R D. Heat transfer from arrays of impinging jets with large jet-to-jet spacing. J Heat Transfer, 1978, 100: 352-357

8 Behbahani A I, Goldstein R J. Local heat transfer to staggered arrays of impinging circular air jets. In: Proceedings of the ASME 1982 International Gas Turbine Conference and Exhibit. London: American Society of Mechanical Engineers, 1982. V004T09A016

9 Qin M, Zheng Q, Ma C F, et al. Experimental studies of local characteristics of heat transfer from a simulated microelectronic chip to an impinging circular dielectric liquid jet (in Chinese). J Eng Thermophys, 1996, 17: 69-74

10 Fabbri M, Dhir V K. Optimized heat transfer for high power electronic cooling using arrays of microjets. J Heat Transfer, 2004, 127: 760769

11 Huang H, Spadaccini L J, Sobel D R. Fuel-cooled thermal management for advanced aeroengines. J Eng Gas Turbines Power, 2004, 126 : 284-293

12 Sobel D R, Spadaccini L J. Hydrocarbon fuel cooling technologies for advanced propulsion. In: Proceedings of the ASME 1995 International Gas Turbine and Aeroengine Congress and Exposition. Houston: American Society of Mechanical Engineers, 1995. V003T06A041

13 Yang V. Modeling of supercritical vaporization, mixing, and combustion processes in liquid-fueled propulsion systems. Proc Combust Institute, 2000, 28: 925-942

14 Zhong F, Fan X, Yu G, et al. Heat transfer of aviation kerosene at supercritical conditions. J Thermophys Heat Transfer, 2009, 23: 543550

15 Gao F, Luo C. Flow-pipe-seepage coupling analysis of spanning initiation of a partially-embedded pipeline. J Hydrodyn, 2010, 22: 478487

16 Wieting A R, Guy R W. Thermal-structural design/analysis of an airframe-integrated hydrogen-cooled scramjet. J Aircraft, 1976, 13: 192-197

17 Zienkiewicz O C, Taylor R L. The Finite Element Method for Solid and Structural Mechanics. 6th ed. Oxford: Elsevier, 2005

18 Kachanov L M. Foundations of the Theory of Plasticity. London: North Holland, 1971

19 China Aeronautical Materials Handbook Editorial Committee. China Aeronautical Materials Handbook (in Chinese). 2nd ed. Beijing: China Standards Press, 2001

20 Webb B W, Ma C F. Single-phase liquid jet impingement heat transfer. In: Hartnett J P, Irvine T F, eds. Advances in Heat Transfer. Elsevier, 1995. 105-217

21 Elison B, Webb B W. Local heat transfer to impinging liquid jets in the initially laminar, transitional, and turbulent regimes. Int $\mathrm{J}$ Heat Mass Transfer, 1994, 37: 1207-1216

22 Xing Y, Spring S, Weigand B. Experimental and numerical investigation of heat transfer characteristics of inline and staggered arrays of impinging jets. J Heat Transfer, 2010, 132: 092201

23 Choo K S, Youn Y J, Kim S J, et al. Heat transfer characteristics of a micro-scale impinging slot jet. Int J Heat Mass Transfer, 2009, 52: 3169-3175

24 Youn Y J, Choo K, Kim S J. Effect of confinement on heat transfer characteristics of a microscale impinging jet. Int J Heat Mass Transfer, 2011, 54: 366-373

25 Pence D V, Boeschoten P A, Liburdy J A. Simulation of compressible micro-scale jet impingement heat transfer. J Heat Transfer, 2003, 125: 447-453

26 Sieder E N, Tate G E. Heat transfer and pressure drop of liquids in tubes. Ind Eng Chem, 1936, 28: 1429-1435 